\title{
The Effect of Common Viral Inactivation Techniques on 16S rRNA Amplicon-Based Analysis of the Gut Microbiota
}

\author{
Zachary McAdams ${ }^{1}\left(\mathbb{D}\right.$, Kevin Gustafson ${ }^{2,3}$ and Aaron Ericsson $2,3,4,5, * \mathbb{D}$ \\ 1 Molecular Pathogenesis and Therapeutics Program, University of Missouri, Columbia, MO 65211, USA; \\ zlmg2b@umsystem.edu \\ 2 Department of Veterinary Pathobiology, University of Missouri, Columbia, MO 65211, USA; \\ klgbkt@missouri.edu \\ 3 Comparative Medicine Program, University of Missouri, Columbia, MO 65211, USA \\ 4 Metagenomics Center, University of Missouri, Columbia, MO 65211, USA \\ 5 Mutant Mouse Resource and Research Center, University of Missouri, Columbia, MO 65211, USA \\ * Correspondence: ericssona@missouri.edu
}

Citation: McAdams, Z.; Gustafson, K.; Ericsson, A. The Effect of Common Viral Inactivation Techniques on 16S rRNA Amplicon-Based Analysis of the Gut Microbiota. Microorganisms 2021, 9, 1755. https://doi.org/10.3390/ microorganisms 9081755

Academic Editor: Didier Hober

Received: 13 July 2021

Accepted: 13 August 2021

Published: 17 August 2021

Publisher's Note: MDPI stays neutral with regard to jurisdictional claims in published maps and institutional affiliations.

Copyright: (c) 2021 by the authors. Licensee MDPI, Basel, Switzerland. This article is an open access article distributed under the terms and conditions of the Creative Commons Attribution (CC BY) license (https:// creativecommons.org/licenses/by/ $4.0 /)$.

\begin{abstract}
Research investigating the gut microbiome (GM) during a viral infection may necessitate inactivation of the fecal viral load. Here, we assess how common viral inactivation techniques affect $16 \mathrm{~S}$ rRNA-based analysis of the gut microbiome. Five common viral inactivation methods were applied to cross-matched fecal samples from sixteen female CD-1 mice of the same GM background prior to fecal DNA extraction. The V4 region of the 16S rRNA gene was amplified and sequenced from extracted DNA. Treatment-dependent effects on DNA yield, genus-level taxonomic abundance, and alpha and beta diversity metrics were assessed. A sodium dodecyl sulfate (SDS)-based inactivation method and Holder pasteurization had no effect on measures of microbial richness, while two Buffer AVL-based inactivation methods resulted in a decrease in detected richness. SDS inactivation, Holder pasteurization, and the AVL-based inactivation methods had no effect on measures of alpha diversity within samples or beta diversity between samples. Fecal DNA extracted with TRIzol-treated samples failed to amplify and sequence, making it unsuitable for microbiome analysis. These results provide guidance in the 16S rRNA microbiome analysis of fecal samples requiring viral inactivation.
\end{abstract}

Keywords: $16 \mathrm{~S}$ rRNA 1; viral inactivation 2; gut microbiome (GM) 3; fecal DNA extraction 4; SDS 5; TRIzol 6; Buffer AVL 7

\section{Introduction}

The gut microbiome (GM) is the diverse community of microorganisms contained within the animal gastrointestinal tract and plays a critical role in host health and physiology [1]. The composition of the GM is dynamic and changes in response to a multitude of factors, including viral infection [2]. Studying the GM during a viral infection is of interest as it may provide further insight into disease progression, disease outcomes, or identification of potential therapeutics. To analyze the GM, the hypervariable regions of the 16S rRNA gene are often amplified from extracted fecal DNA and sequenced; however, handling fecal samples from infected hosts poses a potential risk to the researcher as the samples may harbor infectious viral particles [3,4]. To limit safety risks and comply with appropriate biosafety regulations, the fecal viral load must be inactivated prior to processing and DNA extraction. To our knowledge, the effect of different viral inactivation methods on fecal DNA extraction and targeted amplicon sequencing for GM analysis has not been reported.

Viral inactivation methods can be categorized into three major strategies: pasteurization, detergents, or chemical denaturants. Thermal inactivation is an effective non-invasive technique as it does not require the addition of any reagents [5,6]. In the context of DNA extraction, thermal inactivation methods like Holder pasteurization $\left(63^{\circ} \mathrm{C}\right.$ for $\left.30 \mathrm{~min}\right)$ 
are ideal, as samples are heated at a temperature sufficient for the inactivation of many viral species but remain low enough to maintain DNA integrity for targeted sequence amplification $[7,8]$.

Detergent-based viral inactivation methods frequently utilize sodium dodecyl sulfate (SDS), an anionic detergent that disrupts the membrane of the viral envelope [9,10]. SDS concentrations of $1 \%$ or greater have been shown to be effective at inactivating several viral species $[9,10]$. Moreover, fecal DNA extraction protocols often utilize SDS as a detergent for bacterial cell lysis [11].

Chemical denaturants employ guanidinium thiocyanate as a protein denaturant rendering viruses non-infectious [10,12]. TRIzol Reagent (ThermoFisher Scientific, Waltham, MA, USA) and Buffer AVL (Qiagen, Venlo, the Netherlands) are commercially available nucleic acid extraction reagents that utilize the chaotropic salt as an inactivating agent and are known to be broadly effective against many viral species [10,12]. In addition to guanidinium thiocyanate, TRIzol utilizes phenol as an inactivating agent. Buffer AVL alone is not sufficient to inactivate some viruses and requires an additional pasteurization step to be effective [13].

Here, we simulate viral inactivation in cross-matched fecal samples by applying five common inactivation techniques or reagents-Holder Pasteurization, a SDS lysis buffer, TRIzol Reagent, Buffer AVL, and Buffer AVL with pasteurization-to fecal DNA extraction protocols for targeted 16S rRNA amplification and sequencing. The objective of this work is to assess the treatment-dependent effects of the selected inactivation techniques on DNA yields, measured alpha and beta diversities, and relative taxonomic abundances to guide future $16 \mathrm{~S}$ rRNA amplicon-based analysis of the GM in the context of a viral infection.

\section{Materials and Methods}

\subsection{Sample Collection and Processing}

Freshly evacuated fecal pellets from 16 female CD-1 mice with an Envigo-origin GM (University of Missouri Mutant Mouse Resource \& Research Center, Columbia, MO, USA) [14] were collected simultaneously to minimize temporal changes in the GM. Fecal sample collection was performed by placing a single mouse into an empty autoclaved cage and allowing the mouse to defecate $2-4$ fecal pellets. The mouse was then removed, and autoclaved toothpicks were used to remove the fecal pellets. Following collection, fecal pellets were promptly stored at $-80{ }^{\circ} \mathrm{C}$ until processing. Pellets from each mouse were split into six sections. Each portion was weighed and placed into separate sterile $2 \mathrm{~mL}$ round-bottom tubes. Fecal samples were then stored at $-20{ }^{\circ} \mathrm{C}$ until viral inactivation was simulated, and DNA extracted.

\subsection{Viral Inactivation Simulation and DNA Extraction}

The Qiagen QIAamp PowerFecal Kit (Qiagen, Venlo, The Netherlands) protocol was modified so that samples were mechanically homogenized using a Tissue Lyser II (Qiagen, Venlo, The Netherlands) at $30 \mathrm{~Hz}$ for $10 \mathrm{~min}$ in $2 \mathrm{~mL}$ round-bottom tubes containing a single $0.5 \mathrm{~cm}$ stainless steel bead unless otherwise noted.

\subsubsection{Control Extractions}

DNA from control samples was extracted using the modified Qiagen QIAamp PowerFecal Kit as above.

\subsubsection{SDS}

For SDS-treated samples, DNA was extracted using the ammonium acetate and isopropanol-based method described by Ericsson et al. [11], which utilizes a 4\% SDS lysis buffer. Briefly, fecal samples were mechanically homogenized in $800 \mu \mathrm{L}$ of lysis buffer (500 mM NaCl, $50 \mathrm{mM}$ Tris-HCl, $50 \mathrm{mM}$ EDTA, and 4\% SDS) with a TissueLyser II at $30 \mathrm{~Hz}$ for $3 \mathrm{~min}$ in $2 \mathrm{~mL}$ round-bottom tubes with a single $0.5 \mathrm{~cm}$ stainless steel bead. Following a twenty-minute incubation at $70{ }^{\circ} \mathrm{C}$, samples were centrifuged at $5000 \times g$ for 
$5 \mathrm{~min}$. The supernatant was transferred to a fresh tube and $200 \mu \mathrm{L} 10 \mathrm{mM}$ ammonium acetate were added and mixed by inversion. The samples were incubated on ice for $5 \mathrm{~min}$ then centrifuged at $5000 \times g$ for $5 \mathrm{~min}$. After transferring the supernatant to a fresh tube, an equal volume of cold isopropanol was added. Samples were then incubated on ice for $30 \mathrm{~min}$ before centrifugation at $16,000 \times g$ at $4{ }^{\circ} \mathrm{C}$ for $15 \mathrm{~min}$. The supernatant was discarded, and DNA pellets were washed with $70 \%$ ethanol. DNA was resuspended in $150 \mu \mathrm{L}$ Tris-EDTA (10 mM Tris and $1 \mathrm{mM}$ EDTA) to which $15 \mu \mathrm{L}$ Proteinase $\mathrm{K}$ and $200 \mu \mathrm{L}$ AL buffer from a DNeasy kit (Qiagen, Venlo, The Netherlands) were added. Samples were incubated at $70{ }^{\circ} \mathrm{C}$ for $10 \mathrm{~min}$ before $200 \mu \mathrm{L} 100 \%$ ethanol were added. The sample was transferred to a DNeasy kit spin column and purified following manufacturer instructions.

\subsubsection{Holder Pasteurization}

Samples were briefly centrifuged to collect the pellets to the tube bottom then incubated at $63{ }^{\circ} \mathrm{C}$ for $30 \mathrm{~min}$ [15]. DNA was extracted from the pasteurized pellet using the modified Qiagen QIAamp PowerFecal Kit as above.

\subsubsection{TRIzol}

DNA extractions followed the manufacturer provided TRIzol Reagent (ThermoFisher Scientific, Waltham, MA, USA) Tissue DNA extraction protocol. Briefly, samples were homogenized for $10 \mathrm{~min}$ at $30 \mathrm{~Hz}$ with a TissueLyser II in $1 \mathrm{~mL}$ TRIzol Reagent. Samples were centrifuged at $13,000 \times g$ for $5 \mathrm{~min}$ and the supernatant was transferred to a fresh tube to remove the remaining debris. Samples were incubated at room temperature for $5 \mathrm{~min}$ before $200 \mu \mathrm{L}$ chloroform were added and mixed by vortexing. Samples were incubated at room temperature for $3 \mathrm{~min}$ before centrifugation at $12,000 \times \mathrm{g}$ at $4{ }^{\circ} \mathrm{C}$ for $15 \mathrm{~min}$. The clear aqueous layer was discarded and $300 \mu \mathrm{L}$ absolute ethanol were added. Samples were inverted to mix and incubated for $3 \mathrm{~min}$ at room temperature. The DNA was pelleted at $2000 \times g$ for $5 \mathrm{~min}$ at $4{ }^{\circ} \mathrm{C}$ before discarding the supernatant. The pellet was washed with $1 \mathrm{~mL}$ of $100 \mathrm{mM}$ sodium citrate in $10 \%$ ethanol $(\mathrm{pH} 8.5)$ and incubated at room temperature for $30 \mathrm{~min}$. The DNA was again pelleted and washed with the sodium citrate solution. The DNA was again pelleted, supernatant discarded, then resuspended in $1.5 \mathrm{~mL}$ of $75 \%$ ethanol. The DNA was pelleted once more, supernatant discarded, then allowed to air dry for $10 \mathrm{~min}$. The DNA pellet was resuspended in $8 \mathrm{mM} \mathrm{NaOH}$ by gently pipetting. The sample was centrifuged at $12,000 \times \mathrm{g}$ at $4{ }^{\circ} \mathrm{C}$ for $10 \mathrm{~min}$ before transferring the supernatant to a fresh tube.

\subsubsection{Buffer AVL}

DNA was extracted using a modified Qiagen QIAamp PowerFecal Kit protocol. Briefly, samples were mechanically homogenized in $750 \mu \mathrm{L}$ Buffer AVL (Qiagen, Venlo, The Netherlands) in place of the kit provided PowerBead Solution. The remainder of the extraction protocol followed the modified Qiagen QIAamp PowerFecal Kit protocol as above.

\subsubsection{Buffer AVL with Pasteurization}

DNA was extracted using a modified Qiagen PowerFecal Kit protocol. Samples were briefly vortexed in $750 \mu \mathrm{L}$ Buffer AVL before incubating at $60^{\circ} \mathrm{C}$ for $15 \mathrm{~min}$ [13]. Samples were homogenized as above following pasteurization. The remainder of the extraction protocol followed the modified Qiagen QIAamp PowerFecal Kit protocol as above.

\subsection{7. $16 \mathrm{~S}$ rRNA Amplification and Sequencing}

DNA concentrations of the fecal lysate and purified yields were quantified fluorometrically (Qubit dsDNA BR Assay, Life Technologies, Carlsbad, CA, USA). Samples with concentrations below the limit of detection ( $\mathrm{LOD}, 0.010 \mathrm{ng} / \mathrm{mL}$ ) were recorded as one half of the LOD. Samples were diluted to $3.51 \mathrm{ng} / \mu \mathrm{L}$ before $16 \mathrm{~S}$ rRNA amplification. Samples with a concentration lower than $3.51 \mathrm{ng} / \mu \mathrm{L}$ were concentrated to approximately 
$60 \mu \mathrm{L}$ under vacuum centrifugation at $37^{\circ} \mathrm{C}$. A $60 \mu \mathrm{L}$ aliquot of DNA was submitted for amplification and sequencing for each sample.

The V4 hypervariable region of the 16S rRNA gene was amplified with the dualindexed universal primers (U515F/806R) [16] and flanking Illumina adapters. Each polymerase chain reaction (PCR) contained extracted DNA (up to $100 \mathrm{ng}$ ), U515F/806R universal primers $(0.2 \mu \mathrm{M})$, dNTPs $(200 \mu \mathrm{M})$, and high-fidelity DNA polymerase (Phusion, $1 \mathrm{U})$. The PCR amplification protocol was as follows: $98^{\circ} \mathrm{C}(3: 00)+\left(98^{\circ} \mathrm{C}(0: 15)+50{ }^{\circ} \mathrm{C}(0: 30)\right.$ $\left.+72{ }^{\circ} \mathrm{C}(0: 30)\right) \times 25$ cycles $+72{ }^{\circ} \mathrm{C}(7: 00)$ [11]. The $16 \mathrm{~S}$ rRNA amplicon libraries were pooled and purified with Axygen Axyprep MagPCR clean-up beads. Purified amplicons were diluted to the appropriate concentration for sequencing with an Illumina MiSeq platform using V2 chemistry. $2 \times 250$ bp reads were generated from sequencing.

\subsection{Informatics}

Informatics were performed using QIIME2 v2021.2 [17]. Demultiplexed paired-end reads were trimmed of the universal primers and Illumina adapters using Cutadapt [18]. The trimmed sequences were denoised into amplicon sequence variants (ASVs) with DADA2 and phylogeny was determined using a de novo Mafft FastTree approach [19-21]. Feature tables containing the frequency of unique ASVs observed in each sample were rarefied to 40,000 total features to maximize the proportion of subsampled features (i.e., distinct ASVs) while minimizing the number of discarded samples for diversity analyses [22,23]. Taxonomy for each ASV was assigned with a sklearn feature classifier algorithm using the readytowear $16 \mathrm{~S}$ rRNA 515F-806R SILVA 138 reference database weighted by "animal distal gut" microbial abundances [24-27]. All informatics code can be accessed at https:/ / github.com/ericsson-lab/viral-inactivation.git (accessed on 6 July 2021).

\subsection{Statistical Analysis}

Alpha diversity metrics, one-way permutational analysis of variance (PERMANOVA) tests using Bray-Curtis and Jaccard similarities, fourth-root transformations to normalize data, and principal coordinate analyses ( $\mathrm{PCoA}$ ) were generated with the open-access Past 4.04 software [28]. One-way ANOVA and Tukey HSD post hoc tests were performed with the open-access statistical softwares R v3.6.2 or MetaboAnalyst 5.0 [29,30], with a $p$ value of 0.05 or less considered statistically significant. Three-dimensional PCoAs using Bray-Curtis similarities were generated using the EMPeror plug-in [31] within QIIME2 v2021.2 and visualized at view.qiime2.org (accessed on 6 July 2021).

\section{Results}

\subsection{Not All Viral Inactivation Methods Yield Sequencing-Quality DNA}

DNA concentrations of the fecal lysate and purified yields for each treatment were normalized to the sample mass (Table 1). Significant differences in the fecal lysate DNA concentration between the control extractions and AVL $\left(p=7.8 \times 10^{-3}\right)$, AVL + Heat $(p=0.010)$, and TRIzol $\left(p=5.5 \times 10^{-6}\right)$ groups were observed (Figure S1). Successful 16S rRNA amplification and sequencing were considered to be samples that yielded a per sample sequencing read count greater than 10,000 , as this sequencing depth is sufficient (with similar samples) to detect most rare taxa [11]. Samples yielding less than 10,000 reads were excluded from downstream analysis. While TRIzol-treated samples did yield DNA at concentrations above the LOD, these samples did not produce any successful reads (Table 1). DNA collected from AVL and AVL + Heat inactivation methods frequently yielded DNA concentrations below the LOD but produced completely successful 16S rRNA sequencing data (Table 1). All other samples except one within the control group produced successful read counts. Every TRIzol sample was omitted from downstream analyses as no sample produced successful $16 \mathrm{~S}$ sequencing results. Four additional samples were removed from subsequent analyses after rarefication (Table 1). 
Table 1. Summary of DNA yields, sequencing success, feature count after denoising, and sample number passing the 40,000-feature rarefication filter for each simulated viral inactivation method. Mean \pm SD.

\begin{tabular}{ccccc}
\hline Inactivation Method & $\begin{array}{c}\text { DNA Yield } \\
\text { (ng DNA/mg Feces, } \\
\boldsymbol{n = 1 6 )}\end{array}$ & $\begin{array}{c}\text { Successful 16S } \\
\text { Sequencing } \\
(\geq \mathbf{1 0 , 0 0 0} \text { Reads) }\end{array}$ & $\begin{array}{c}\text { Features per Sample } \\
\text { Post-Denoising } \\
\text { (Mean } \pm \text { SD, } \boldsymbol{n = 1 6 )}\end{array}$ & $\begin{array}{c}\text { Sample Number } \\
\text { Passing 40,000 Feature } \\
\text { Rarefication Filter }\end{array}$ \\
\hline Control & $135.9 \pm 106.2$ & $15 / 16$ & $99,572 \pm 34,364$ & $15 / 16$ \\
SDS & $242.0 \pm 186.0$ & $16 / 16$ & $84,754 \pm 17,681$ & $15 / 16$ \\
TRIzol & $18.7 \pm 19.3$ & $0 / 16$ & 0 & $0 / 16$ \\
Holder & $65.3 \pm 29.5$ & $16 / 16$ & $111,909 \pm 17,831$ & $16 / 16$ \\
AVL & $0.2 \pm 0.5$ & $16 / 16$ & $62,508 \pm 18,162$ & $15 / 16$ \\
AVL + Heat & $0.4 \pm 0.8$ & $16 / 16$ & $72,796 \pm 21,625$ & $14 / 16$ \\
\hline
\end{tabular}

\subsection{Treatment-Dependent Effects on Microbial Richness but Not Distribution or Beta Diversity}

Data were assessed for treatment-dependent effects on alpha diversity measures of richness and distribution. No significant differences were detected in the observed or predicted richness between samples in the SDS and Holder pasteurization groups comparted to the control (Figure 1A,B); however, significant differences in both the observed $\left(p=2.0 \times 10^{-3}\right)$ and predicted $\left(p=7 \times 10^{-4}\right)$ richness were detected in samples subjected to AVL inactivation. A significant difference in the predicted richness between AVL + Heat inactivation and control was observed $(p=0.021)$. No significant differences in the distribution of features within samples using Simpson or Shannon indices were observed between inactivation methods (Figure 1C,D).

A

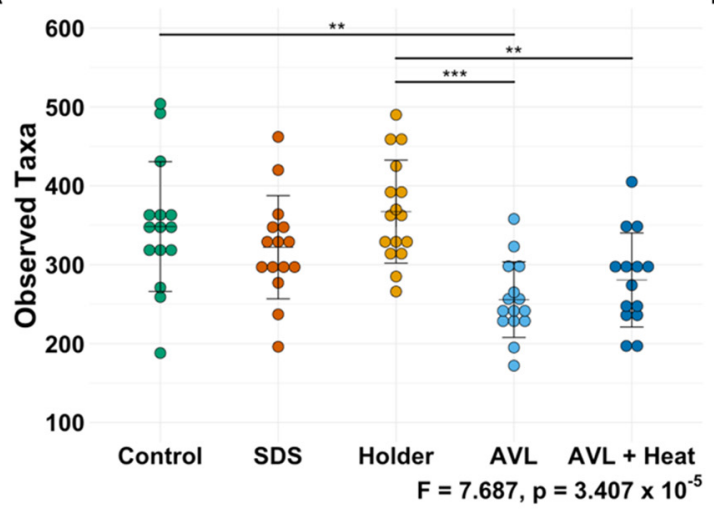

C

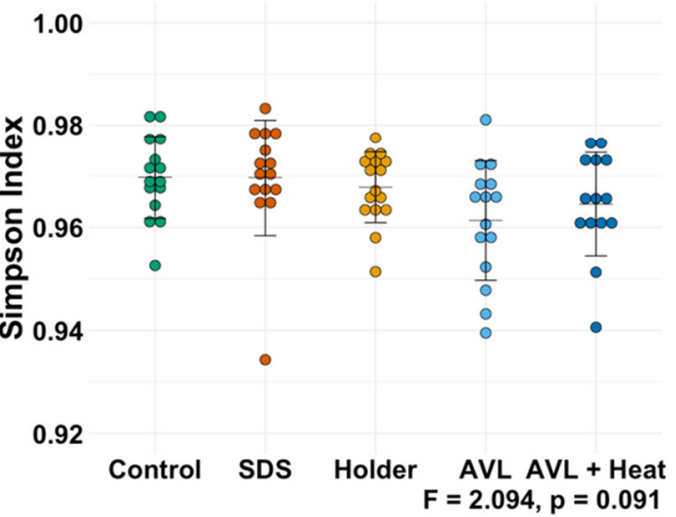

B

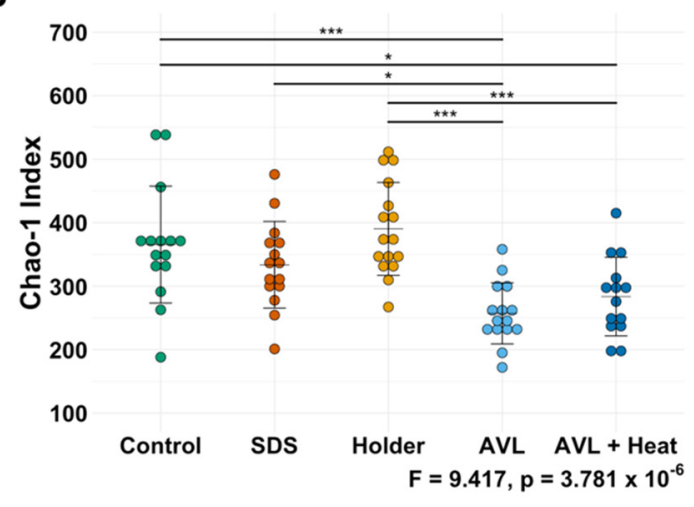

D

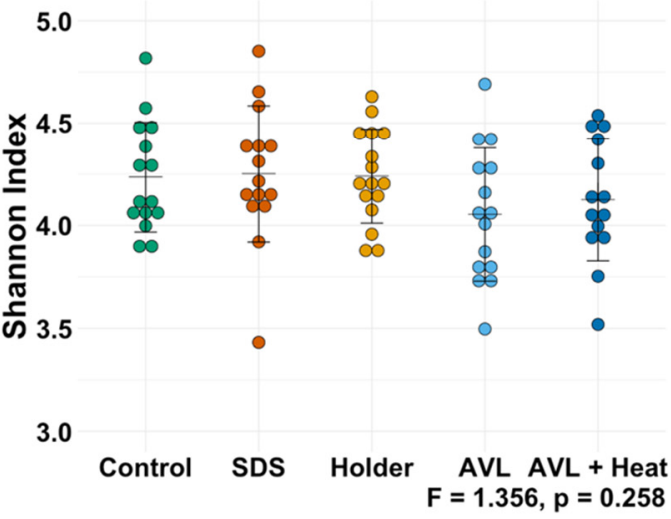

Figure 1. Alpha diversity metrics per viral inactivation method: (A) Observed Taxa, (B) Chao-1 Index, (C) Simpson Index, (D) Shannon Index; Control ( $n=15)$, SDS ( $n=15)$, TRIzol (omitted), Holder $(n=16)$, AVL $(n=15)$, AVL + Heat $(n=14)$. Dots represent individual data points, bars represent mean \pm SD, one-way ANOVA followed by Tukey HSD post-hoc test; ${ }^{*} p<0.05,{ }^{* *} p<0.01,{ }^{* * *} p<0.001$. 
Next, the relative abundance of taxa at the genus level within each sample from individual donors was compared to subjectively assess the effect of inactivation method on detected taxonomy (Figure 2). Only 5 of the 120 detected genera were found to be significantly different between inactivation methods, suggesting little effect of the inactivation method on determined taxonomy (Supplementary Table S1). To objectively assess the effect of inactivation on beta diversity between samples, two-dimensional PCoAs of the rarefied feature table with a fourth-root transformation to normalize data showed a high similarity between all inactivation methods using both weighted (Bray-Curtis, $p=0.21, \mathrm{~F}=1.172$ ) and unweighted (Jaccard, $p=0.39, \mathrm{~F}=1.019$ ) metrics (Figure 3). Pairwise comparisons revealed a significant, albeit subtle, difference between the AVL and Holder inactivation groups using Jaccard similarities ( $p=0.044, \mathrm{~F}=1.609)$.

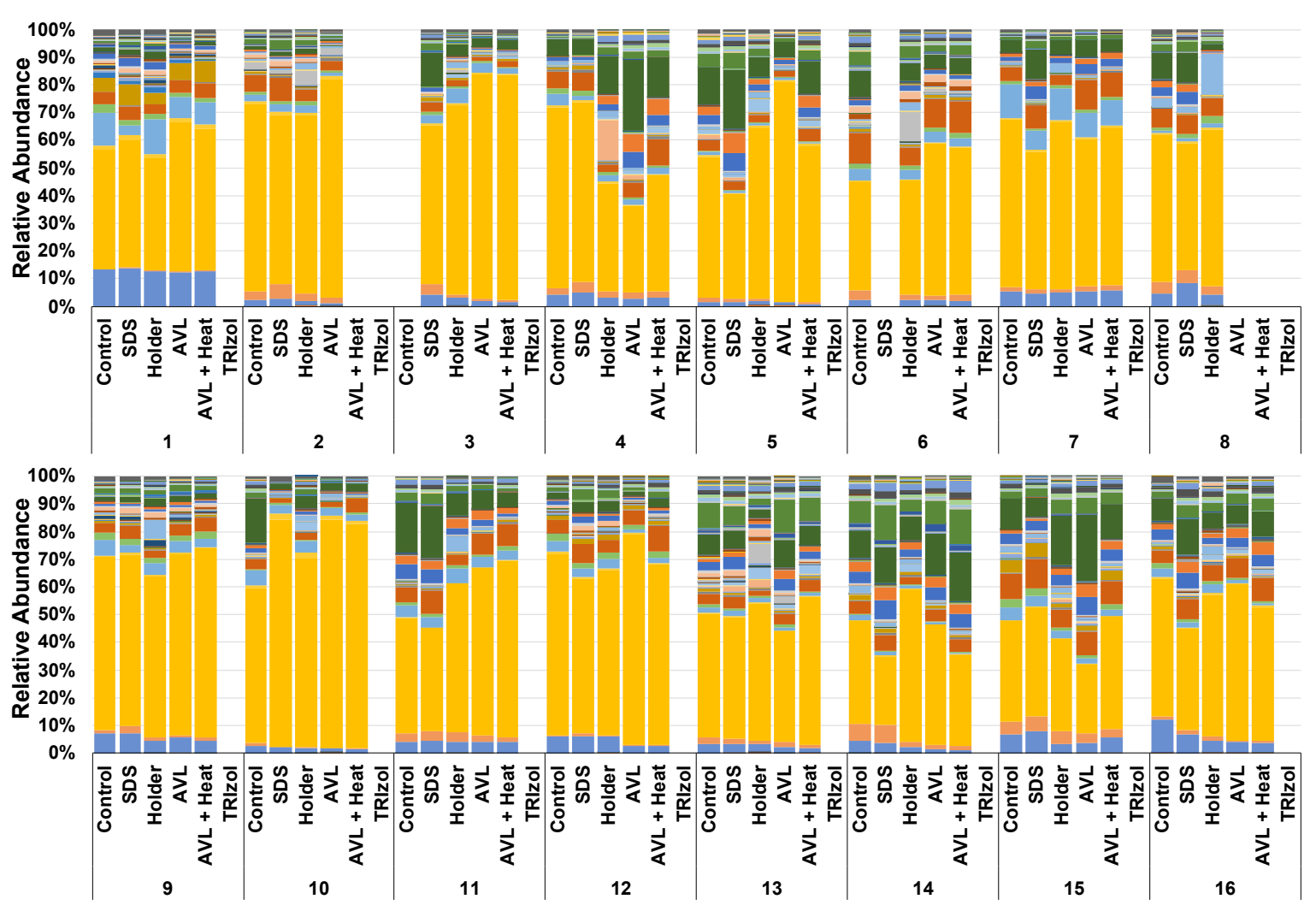

Figure 2. Stacked bar chart displaying per sample relative taxonomic abundance at the genus level. Samples are organized by sample donor. Treatments labels with no bar indicate the samples either did not successfully sequence or did not pass the rarefaction filter.

A

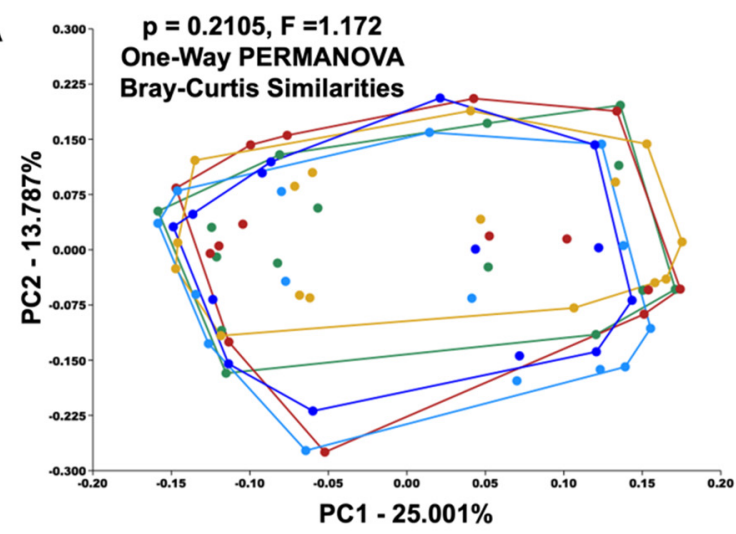

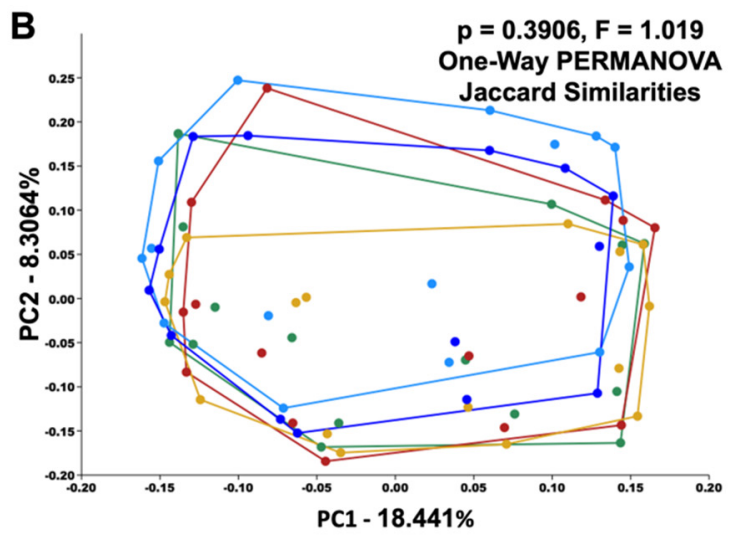

Control

SDS

Holder

AVL

AVL + Heat

Figure 3. Two-dimensional principal coordinate analysis of (A) Bray-Curtis and (B) Jaccard similarities; Control ( $n=15)$, SDS $(n=15)$, TRIzol (omitted), Holder $(n=16)$, AVL $(n=15)$, AVL + Heat $(n=14)$; one-way PERMANOVA. 
Consistent with the two-dimensional PCoAs, three-dimensional PCoA plots showed no clustering of samples when labeled by inactivation method but did demonstrate clustering when samples were labeled by donor using both weighted (Figure S2A,B) and unweighted metrics (Figure S2C,D). Interactive three-dimensional PCoAs are available for download at https:/ / github.com/ericsson-lab/viral-inactivation.git (accessed on 6 July 2021) and are viewable at view qiime2.org (accessed on 6 July 2021).

\section{Discussion}

While several studies have tested the efficacy of these and other inactivation methods against numerous viruses, the current study was designed to assess the influence of several methods of viral inactivation on the suitability of residual DNA in the fecal matrix for use as templates in downstream targeted amplicon sequencing. These methods were selected based on feasibility for a researcher working in high BSL conditions to inactivate the fecal viral load before transporting samples to a facility of the appropriate biosafety level for DNA extraction, and for their likelihood of preserving DNA integrity of the samples. These methods are not effective against all virus types, or in all sample matrices, making it important for researchers to validate that their virus of interest can be inactivated in fecal biomass by one of these methods before DNA is extracted for 16S rRNA library generation and sequencing.

Our data indicate that the use of a surfactant (SDS)-based lysis buffer or heat-based inactivation (Holder pasteurization) have no detectable effect on final assessments of alpha or beta diversity, making them preferable viral inactivation techniques for samples intended for $16 \mathrm{~S}$ rRNA analysis, assuming they are effective against the virus in question. Guanidinium-based buffers (i.e., TRIzol and AVL) produced mixed results. While TRIzol is highly effective in the inactivation of many viruses within the Alphavirus, Bunyavirus, Filovirus, Flavivirus, Simplexvirus, Adenovirus, Enterovirus, Influenzavirus, and Coronavirus (e.g., SARS-CoV2) genera [10,12,32], it is not supported by the current data as a means of viral inactivation of samples intended for targeted amplicon sequencing. Fecal DNA extracted with TRIzol may require further purification to remove residual amplification or sequencing inhibitors, despite detectable, albeit reduced, DNA yields.

It is unclear why the methods including AVL (either with or without heat) resulted in such low DNA yields. It is also notable that, despite those low yields, the AVL-inactivated samples were still able to provide meaningful data regarding beta diversity, although alpha diversity was negatively affected. It is unknown however whether the observed differences were due to a sequence bias exhibited by the buffer during extraction or if rarefying a greater proportion of total features favored lower measured richness, as rare taxa may not be represented (Table 1). Regardless, these data would suggest that, while not optimal, AVL-inactivated fecal samples could still yield meaningful targeted amplicon sequencing data, assuming all samples were inactivated in an identical manner. Like TRIzol, it is possible that AVL-based methods could be optimized with additional purification steps. Regardless, when considering DNA yields and sequencing data collectively, SDS- and heat-based inactivation protocols are preferable platforms for fecal samples intended for targeted amplicon sequencing. Collectively, these data will help guide the selection of a method for fecal viral inactivation for $16 \mathrm{~S}$ rRNA amplicon-based microbiome analysis.

Supplementary Materials: The following are available online at https: / www.mdpi.com/article / 10.3390/microorganisms9081755/s1, Figure S1: Treatment-dependent differences in fecal lysate DNA concentration normalized to pellet mass, Figure S2: Three-dimensional PCoA plots of samples labelled by inactivation method and sample donor using weighted and unweighted metrics. Table S1: Summary of genera exhibiting significantly different abundances between inactivation methods.

Author Contributions: Conceptualization, A.E.; Data curation, Z.M.; Formal analysis, Z.M. and A.E.; Funding acquisition, A.E.; Investigation, Z.M. and K.G.; Methodology, Z.M., K.G. and A.E.; Visualization, Z.M.; Writing—original draft, Z.M.; Writing—review \& editing, Z.M., K.G. and A.E. All authors have read and agreed to the published version of the manuscript. 
Funding: This work was supported by the University of Missouri Metagenomics Center.

Institutional Review Board Statement: The study was conducted according to the guidelines of the Institutional Animal Care and Use Committee of the University of Missouri (protocol 9587).

Data Availability Statement: Sequences can be accessed at the National Center for Biotechnology Information, Sequence Read Archive (SRA) with the BioProject ID PRJNA71299. All code used to process 16S rRNA sequences and interactive PCoA plots can be accessed at: https:/ / github.com/ ericsson-lab/viral-inactivation.git (accessed on 6 July 2021).

Acknowledgments: The authors would like to acknowledge the University of Missouri DNA Core for their work generating and sequencing $16 \mathrm{~S}$ rRNA amplicon libraries.

Conflicts of Interest: The authors declare no conflict of interest.

\section{References}

1. Liang, D.; Leung, R.K.-K.; Guan, W.; Au, W.W. Involvement of Gut Microbiome in Human Health and Disease: Brief Overview, Knowledge Gaps and Research Opportunities. Gut Pathog. 2018, 10, 3. [CrossRef] [PubMed]

2. Li, N.; Ma, W.-T.; Pang, M.; Fan, Q.-L.; Hua, J.-L. The Commensal Microbiota and Viral Infection: A Comprehensive Review. Front. Immunol. 2019, 10, 1551. [CrossRef] [PubMed]

3. Wang, X.; Zheng, J.; Guo, L.; Yao, H.; Wang, L.; Xia, X.; Zhang, W. Fecal Viral Shedding in COVID-19 Patients: Clinical Significance, Viral Load Dynamics and Survival Analysis. Virus Res. 2020, 289, 198147. [CrossRef] [PubMed]

4. Koçer, Z.A.; Obenauer, J.; Zaraket, H.; Zhang, J.; Rehg, J.E.; Russell, C.J.; Webster, R.G. Fecal Influenza in Mammals: Selection of Novel Variants. J. Virol. 2013, 87, 11476-11486. [CrossRef]

5. $\quad$ Park, S.L.; Huang, Y.-J.S.; Hsu, W.-W.; Hettenbach, S.M.; Higgs, S.; Vanlandingham, D.L. Virus-Specific Thermostability and Heat Inactivation Profiles of Alphaviruses. J. Virol. Methods 2016, 234, 152-155. [CrossRef] [PubMed]

6. Hamprecht, K.; Maschmann, J.; Müller, D.; Dietz, K.; Besenthal, I.; Goelz, R.; Middeldorp, J.M.; Speer, C.P.; Jahn, G. Cytomegalovirus (CMV) Inactivation in Breast Milk: Reassessment of Pasteurization and Freeze-Thawing. Pediatr. Res. 2004, 56, 529-535. [CrossRef] [PubMed]

7. Batéjat, C.; Grassin, Q.; Manuguerra, J.-C.; Leclercq, I. Heat Inactivation of the Severe Acute Respiratory Syndrome Coronavirus 2. J. Biosaf. Biosecur. 2021, 3, 1-3. [CrossRef]

8. García-González, I.; Corona-Cervantes, K.; Hernández-Quiroz, F.; Villalobos-Flores, L.E.; Galván-Rodríguez, F.; Romano, M.C.; Miranda-Brito, C.; Piña-Escobedo, A.; Borquez-Arreortúa, F.G.; Rangel-Calvillo, M.N.; et al. The Effect of Holder Pasteurization on the Diversity of the Human Milk Bacterial Microbiota Using High-Throughput DNA Sequencing. J. Hum. Lact. 2021, 089033442110119. [CrossRef]

9. Urdaneta, S.; Wigdahl, B.; Neely, E.B.; Berlin, C.M.; Schengrund, C.-L.; Lin, H.-M.; Howett, M.K. Inactivation of HIV-1 in Breast Milk by Treatment with the Alkyl Sulfate Microbicide Sodium Dodecyl Sulfate (SDS). Retrovirology 2005, 2, 28. [CrossRef]

10. Patterson, E.I.; Prince, T.; Anderson, E.R.; Casas-Sanchez, A.; Smith, S.L.; Cansado-Utrilla, C.; Solomon, T.; Griffiths, M.J.; Acosta-Serrano, Á.; Turtle, L.; et al. Methods of Inactivation of SARS-CoV-2 for Downstream Biological Assays. J. Infect. Dis. 2020, 222, jiaa507. [CrossRef]

11. Ericsson, A.C.; Davis, J.W.; Spollen, W.; Bivens, N.; Givan, S.; Hagan, C.E.; McIntosh, M.; Franklin, C.L. Effects of Vendor and Genetic Background on the Composition of the Fecal Microbiota of Inbred Mice. PLoS ONE 2015, 10, e0116704. [CrossRef]

12. Blow, J.A.; Dohm, D.J.; Negley, D.L.; Mores, C.N. Virus Inactivation by Nucleic Acid Extraction Reagents. J. Virol. Methods 2004, 119, 195-198. [CrossRef]

13. Smither, S.J.; Weller, S.A.; Phelps, A.; Eastaugh, L.; Ngugi, S.; O’Brien, L.M.; Steward, J.; Lonsdale, S.G.; Lever, M.S. Buffer AVL Alone Does Not Inactivate Ebola Virus in a Representative Clinical Sample Type. J. Clin. Microbiol. 2015, 53, 3148-3154. [CrossRef]

14. Hart, M.L.; Ericsson, A.C.; Lloyd, K.C.K.; Grimsrud, K.N.; Rogala, A.R.; Godfrey, V.L.; Nielsen, J.N.; Franklin, C.L. Development of Outbred CD1 Mouse Colonies with Distinct Standardized Gut Microbiota Profiles for Use in Complex Microbiota Targeted Studies. Sci. Rep. 2018, 8, 10107. [CrossRef]

15. Pitino, M.A.; O'Connor, D.L.; McGeer, A.J.; Unger, S. The Impact of Thermal Pasteurization on Viral Load and Detectable Live Viruses in Human Milk and Other Matrices: A Rapid Review. Appl. Physiol. Nutr. Metab. 2020, 99, 1-17. [CrossRef]

16. Caporaso, J.G.; Lauber, C.L.; Walters, W.A.; Berg-Lyons, D.; Lozupone, C.A.; Turnbaugh, P.J.; Fierer, N.; Knight, R. Global Patterns of 16S RRNA Diversity at a Depth of Millions of Sequences per Sample. Proc. Natl. Acad. Sci. USA 2011, 108, 4516-4522. [CrossRef] [PubMed]

17. Bolyen, E.; Rideout, J.R.; Dillon, M.R.; Bokulich, N.A.; Abnet, C.C.; Al-Ghalith, G.A.; Alexander, H.; Alm, E.J.; Arumugam, M.; Asnicar, F.; et al. Reproducible, Interactive, Scalable and Extensible Microbiome Data Science Using QIIME 2. Nat. Biotechnol. 2019, 37, 852-857. [CrossRef] [PubMed]

18. Martin, M. Cutadapt Removes Adapter Sequences from High-Throughput Sequencing Reads. EMBnet J. 2011, 17, 10-12. [CrossRef]

19. Callahan, B.J.; McMurdie, P.J.; Rosen, M.J.; Han, A.W.; Johnson, A.J.A.; Holmes, S.P. DADA2: High-Resolution Sample Inference from Illumina Amplicon Data. Nat. Methods 2016, 13, 581. [CrossRef] [PubMed] 
20. Price, M.N.; Dehal, P.S.; Arkin, A.P. FastTree 2-Approximately Maximum-Likelihood Trees for Large Alignments. PLoS ONE 2010, 5, e9490. [CrossRef]

21. Katoh, K.; Standley, D.M. MAFFT Multiple Sequence Alignment Software Version 7: Improvements in Performance and Usability. Mol. Biol. Evol. 2013, 30, 772-780. [CrossRef]

22. Weiss, S.; Xu, Z.Z.; Peddada, S.; Amir, A.; Bittinger, K.; Gonzalez, A.; Lozupone, C.; Zaneveld, J.R.; Vázquez-Baeza, Y.; Birmingham, A.; et al. Normalization and Microbial Differential Abundance Strategies Depend upon Data Characteristics. Microbiome 2017, 5, 27. [CrossRef] [PubMed]

23. McDonald, D.; Clemente, J.C.; Kuczynski, J.; Rideout, J.R.; Stombaugh, J.; Wendel, D.; Wilke, A.; Huse, S.; Hufnagle, J.; Meyer, F.; et al. The Biological Observation Matrix (BIOM) Format or: How I Learned to Stop Worrying and Love the Ome-Ome. Gigascience 2012, 1, 7. [CrossRef] [PubMed]

24. Kaehler, B.D.; Bokulich, N.A.; McDonald, D.; Knight, R.; Caporaso, J.G.; Huttley, G.A. Species Abundance Information Improves Sequence Taxonomy Classification Accuracy. Nat. Commun. 2019, 10, 4643. [CrossRef]

25. Bokulich, N.A.; Kaehler, B.D.; Rideout, J.R.; Dillon, M.; Bolyen, E.; Knight, R.; Huttley, G.A.; Caporaso, J.G. Optimizing Taxonomic Classification of Marker-Gene Amplicon Sequences with QIIME 2's Q2-Feature-Classifier Plugin. Microbiome 2018, 6, 90. [CrossRef] [PubMed]

26. Quast, C.; Pruesse, E.; Yilmaz, P.; Gerken, J.; Schweer, T.; Yarza, P.; Peplies, J.; Glöckner, F.O. The SILVA Ribosomal RNA Gene Database Project: Improved Data Processing and Web-Based Tools. Nucleic Acids Res. 2013, 41, D590-D596. [CrossRef] [PubMed]

27. Robeson, M.S.; O’Rourke, D.R.; Kaehler, B.D.; Ziemski, M.; Dillon, M.R.; Foster, J.T.; Bokulich, N.A. RESCRIPt: Reproducible Sequence Taxonomy Reference Database Management for the Masses. Biorxiv 2020. [CrossRef]

28. Hammer, Ø.; Harper, D.A.T.; Ryan, P.D. PAST: Paleontological Statistics Software Package for Education and Data Analysis. Palaeontol. Electron. 2001, 4, 9.

29. RC Team. R: A Language and Environment for Statistical Computing; RC Team: Vienna, Austria, 2019.

30. Pang, Z.; Chong, J.; Zhou, G.; de Lima Morais, D.A.; Chang, L.; Barrette, M.; Gauthier, C.; Jacques, P.-É.; Li, S.; Xia, J. MetaboAnalyst 5.0: Narrowing the Gap between Raw Spectra and Functional Insights. Nucleic Acids Res. 2021, 49, gkab382. [CrossRef]

31. Vázquez-Baeza, Y.; Pirrung, M.; Gonzalez, A.; Knight, R. EMPeror: A Tool for Visualizing High-Throughput Microbial Community Data. Gigascience 2013, 2, 16. [CrossRef]

32. Ngo, K.A.; Jones, S.A.; Church, T.M.; Fuschino, M.E.; George, K.S.; Lamson, D.M.; Maffei, J.; Kramer, L.D.; Ciota, A.T. Unreliable Inactivation of Viruses by Commonly Used Lysis Buffers. Appl. Biosaf. 2017, 22, 56-59. [CrossRef] 